\title{
In Vitro Rigidity Effect on Breast Cancer MCF-7 Three- Dimensional Microtissue Grown from Few Cells
}

\author{
Afrimzon Elena ${ }^{1}$, Moshkov Sergei ${ }^{1}$, Shafran Yana ${ }^{1}$, Pelov Ronen ${ }^{2}$, Freund Maya ${ }^{3}$, Hakuk Yaron ${ }^{1}$, \\ Bar-On-Eizig Zehavit ${ }^{1}$, Zurgil Naomi ${ }^{1}$ and Deutsch Mordechai ${ }^{1 *}$ \\ ${ }^{1}$ Department of Physics, Bar-Ilan University, Israel \\ ${ }^{2}$ Rhenium LTD, Israel \\ ${ }^{3}$ The National Center of Forensic Medicine, Israel
}

*Corresponding author: Mordechai Deutsch, The Biophysical Interdisciplinary Schottenstein Center for the Research and Technology of the Cellome, Department of Physics Building 214, Bar-Ilan University, Ramat Gan, 5290002, Israel.

To Cite This Article: Afrimzon E, Moshkov S, Shafran Y, Pelov R, Freund M, Hakuk Y, Bar-On-Eizig Z, Zurgil N and Deutsch M. In Vitro Rigidity Effect on Breast Cancer MCF-7 Three-Dimensional Microtissue Grown from Few Cells. Am J Biomed Sci \& Res. 2019 - 1(2). AJBSR.MS.ID.000515. DOI: 10.34297/AJBSR.2019.01.000515

Received: January 10, 2019 | Published: January 23, 2019

\section{Abstract}

Background: Biological tissues normally possess varying rigidities, changes in which may reflect transformation from normal to pathological state. Cancer cells within the tumor are influenced by the mechanical conditions of their microenvironment, which can drive cell fate.

Methods: We present a new on-array methodology to mimic and control desired surrounding rigidity in vitro for three-dimensional (3D) breast cancer object formation and growth. 3D objects were generated from single cells within a hydrogel array, cultured under various mechanical conditions and measured at single-object resolution.

Results: Alterations in development of 3D breast cancer microtissue under various rigidity conditions in vitro are revealed. Object growth rate, morphology and vital features are associated with the extent of environmental rigidity, the point in time at which embedding was performed and the initial number of seeded cells. Under stiffness that resembles tumor tissue, higher growth rate of breast cancer microtissue and specific "preinvasive" phenotype are evident. Hallmarks of this phenotype include loose morphology with unclear edges and massive peripheral cell spread, dispersed intrinsic structure and reduced expression of epithelial surface markers

Conclusions: Physical changes in cell environment without parallel changes in biochemical conditions affect growth and development of 3D cancer microtissue grown from individual cells in vitro. A stiffer environment supports 3D microtissue growth and its morphologic and functional diversity in comparison to regular low rigidity conditions.

Keywords: Breast Cancer, Image Analysis, Environmental Carcinogens, Agarose, Proof of Concept

Abbreviations: LMA: Low Melting Agarose; PI: Propidium Iodide; TMRM: Tetramethylrhodamine Methyl Ester; PBS: Phosphate Buffer Saline; hMCA: Hydrogel Microchamber Array; MCs: Microchambers; H\&E: Hematoxylin-Eosin; BF: Bright Field; ROI: Region of Interest; SA: Sectional Area; FI: Fluorescence Intensity; VM algorithm: Support Vector Machine Algorithm; K-S test: Kolmogorov-Smirnov test

\section{Introduction}

Biological tissues normally possess different rigidities which contribute to its physiological function. Live cells recognize environmental stiffness that triggers signaling pathways, resulting in changes to cell morphology, spread and movement, including trafficking via basement membrane [1,2]. Moreover, rigidity is one of the regulatory mechanisms in the organization/reorganization of developing tissue [3], in cell proliferation, differentiation and consequently, apoptosis, cell adhesion, polarity, contractility and migration, as well as protein expression, secretion and metabolic activity [4,5]. Cells and extracellular components of mature biological tissues generally maintain a dynamic balance, which, when fails, causes anomalies that promote progression of disease, including cancer [6]. Changes in tissue rigidity may reflect tissue transformation from normal to pathological state $[7,8]$. Increased 
tissue rigidity of primary breast tumors correlates with metastatic recurrence and poor patient survival [9].

Solid tumors develop in vivo, interact with and modify the local conditions, including microenvironmental stiffness, in favor of tumor progression [10,11]. Cancer cells within the tumor are intimately influenced by mechanical conditions, biochemical signals and cell-cell interactions which can change its genetic profile [12] and tumor tissue morphology [13,14], as well as driving its fate $[15,16]$. Culturing under altered stiffness conditions without simultaneous biochemical impact, indicates that tension can drive tumor progression through destabilization of adherent junctions that can act as cellular mechanosensors, whereas disseminated tumor cells may respond to mechanical cues differently until they re-epithelialize [9].

The last twenty years have seen in vitro research shift towards three-dimensional (3D) cell culture models which are recognized as superior to the commonly used 2D models, as they better mimic the natural structure in vivo, thereby providing a more accurate in vivo-like Organization and response to external stimuli. Today, it is believed that different tissue culture conditions, 3D as opposed to $2 \mathrm{D}$, affect the physical, chemical/biochemical and biological stimuli and induce changes in cell signaling at multiple levels, such as transduction, transcription and post-transcription, cell phenotypes, growth, invasion and survival under anti-cancer drug treatment [17].

Cancer cells grown as multicellular 3D structures demonstrate the ability to quickly adapt response to extracellular stimuli, resulting in higher resistance to treatment.

Special conditions are required for 3D culturing of epithelial cells in vitro, wherein cell-cell adhesion is stronger than cell adhesion to substrates. Currently, polymer or natural hydrogels, such as agarose are the most widely used non-adherent substrates. Agarose forms a macroporous matrix which allows rapid diffusion of molecules including macromolecules unrestricted by the gel [18]. Agarose, being nontoxic to living cells, is used for cell investigation, including cell migration study [19], bio mimetics of vasculature [20], creation of synthetic analogs of basement membrane [21], and as a half-liquid medium for cellular 3D structure formation [16,22]. Additionally, its rheological properties make it useful for modulation of matrix stiffness in vitro [23]. Finally, optical properties of agarose gel are excellent for live-cell visualization and observation due to its refractive index being comparable to water $[24,25]$.

Manipulating the mechanical feature of microenvironments in which cancer cells interact with surrounding stiffness in 3D cell cultures remains challenging. Development of the optimal mechanical conditions in vitro can benefit both the scientific study of cancer tumor evaluation and biomedical applications, including anti-cancer drug screening, stem cell study, regenerative medicine, biomedical and tissue engineering.

In this study we propose an efficient approach to reach desired surrounding rigidity in vitro for 3D breast cancer model formation and growth. The excellent mechanical and optical Properties of agarose were used for the measurements of arrayed non-adherent non-tethered 3D objects under different mechanical conditions at single-spheroid resolution. Three-dimensional microtissues were generated from single cells in a hydrogel array, cultured under various mechanical conditions which were created by the process of agarose embedding, and measured at single-object resolution. The study demonstrates alteration in 3D breast cancer microtissue creation, formation and development in vitro under the influence of various stiffness conditions at individual 3D object level. Significant differences that are associated with the extent of environmental rigidity, the point in time at which embedding was performed and the initial number of seeded cells, were revealed in microtissue growth rate, morphology and vital characteristics. The 3D objects initiated from less than eight cells are significantly different from those initiated by more cells and demonstrate a growth rate independent from surrounding rigidity. Under stiffness conditions that resemble tumor tissue, a higher growth rate of breast cancer microtissues and specific "pre-invasive" phenotype were evident. In contrast, the subset with the specific "pre-invasive" phenotype has not been observed in the control culture of 3D objects grown freely under low-rigidity conditions.

\section{Materials and Methods}

\section{Materials}

Low melting agarose (LMA) was obtained from Cambrex Bio Science Rockland, Inc. (Rockland, ME USA). Sylgard 184 Kit was purchased from Dow Corning Corp. (Midland, MI, USA). Propidium iodide (PI) and tetramethylrhodamine methyl ester (TMRM) were obtained from Sigma-Aldrich (St.Louis, MO, USA). Fluorescein diacetate (FDA) was purchased from Sigma-Riedel-de-Haen (Hannover, Germany). Monoclonal antibodies against EpCam and CXCR4 markers were purchased from Miltenyi Biotec (Bergisch Gladbach, Germany). DMEM medium, heat-inactivated fetal calf serum (FCS), penicillin, streptomycin, glutamine, sodium pyrovate and phosphate buffer saline (PBS) were obtained from Biological Industries (Kibbutz Beit Haemek, Israel). Six-well glass bottom plates were purchased from In Vitro Scientific (Sunnyvale, CA, USA).

\section{Methods}

The Hydrogel Microchamber Array (hMCA) was designed and fabricated as described previously [26]. Briefly, an array of square bottom pyramid shaped microchambers (MCs) was obtained from GeSiM mbH (Großerkmannsdorf, Germany) and used for production of the PDMS stamp with a negative MC array. Fabrication of the 
hMCA was performed in the specially modified commercial six-well glass bottom plates. Warm LMA was dripped on the surface of the plate's glass bottom and pre-heated PDMS stamp gently placed over it. The system was incubated at RT for 5-7 min for pre-gelling and pre-cooling, followed by $10 \mathrm{~min}$ incubation at $4^{\circ} \mathrm{C}$ for LMA gelation. At the culmination of the gelation process, the PDMS stamp was peeled off, leaving agarose gel patterned with square geometry MCs (from 400 to 800 in each macro well) with $90 \mu \mathrm{m}$ on each side and about $100 \mu \mathrm{m}$ depth. The imaging plate, consisting of optical bottom patterned with hMCA was UV sterilized and stored at $4^{\circ} \mathrm{C}$ in humidified atmosphere until use.

Measurement of hydrogel mechanical properties: The storage and loss shear moduli, as well as complex shear moduli of the agarose hydrogels were measured using an MCR 102 Modular Compact Rheometer (Anton Paar GmbH, Austria). The experiment was conducted using a fixed roughened bottom plate of $50 \mathrm{~mm}$ diameter and a top roughened rotating plate of $25 \mathrm{~mm}$ diameter within the linear viscoelastic region of the samples (from 0.1 to $100 \%$ ) at a frequency of $1.0 \mathrm{~Hz}$ at $37^{\circ} \mathrm{C}$.

Cell line and culture: MCF-7 Human Breast Cancer cells were maintained in DMEM medium, supplemented with $10 \%$ heat-inactivated fetal calf serum, $100 \mathrm{U} / \mathrm{mL}$ penicillin, $100 \mu \mathrm{g} /$ $\mathrm{mL}$ streptomycin, $2 \%$ glutamine, $2 \%$ sodium pyruvate (complete medium). Cells were maintained in completely humidified atmosphere with $5 \% \mathrm{CO}_{2}$ at $37^{\circ} \mathrm{C}$. Before use, the exponentially growing cells were collected by trypsinization, washed and resuspended at appropriate concentrations in fresh complete medium.

Cellular microtissue formation: Cell suspension $(50 \mu \mathrm{L}, 0.1$ $0.3 \times 10^{6}$ cells $/ \mathrm{mL}$ ) was loaded onto hMCA, and then set aside to allow cellular/multicellular structure formation in each MC. Loaded MCF-7 cells were embedded in agarose gel with different w/v concentrations $(1 \%, 2 \%$ or $3 \%)$ at $19 \mathrm{~h}$ or immediately after cell loading to create suitable mechanical conditions. The 3D spheroids grown either in the hydrogel layer or freely (control), each in its individual MC, were monitored for seven days.

Experimental scheme: Cells were loaded into agarose MCs and imaged (time point zero). They were embedded in the freshly prepared agarose hydrogel immediately after loading or after 19 $\mathrm{h}$ of incubation under standard conditions (completely humidified atmosphere with $5 \% \mathrm{CO}_{2}$ at $37^{\circ} \mathrm{C}$ ) by the procedure of the medium exchange with hydrogel. For this procedure, the heated agarose solution was previously cooled to $37^{\circ} \mathrm{C}$ and then introduced into the chip before gelation. At this stage, agarose solution easily filled the MCs and surrounded the loaded cells or initial cellular conglomerates which resulted from cell embedding. Next, the "sandwich" chip was allowed to cool for a few minutes at $4^{\circ} \mathrm{C}$ until the agarose gelled. After this, the fresh complete medium at RT was added into the device. In the control samples, the medium was exchanged with fresh medium (agarose $0 \%$ ) at the appropriate time points and treated at $4{ }^{\circ} \mathrm{C}$, respectively. Spheroid formation and growth were prolonged until 7 days under regular conditions with medium being exchanged twice during the experiment. Image acquisition was performed continuously at each experimental step.

\section{Cellular object staining}

All live fluorescent staining procedures and post- fixation staining were performed in situ while multicellular microstructures were still within the hMCA. For adequate control staining and measurements, control spheroids grown freely were embedded in agarose right before staining. Cytoplasm membrane integrity and cellular microtissue viability were determined by double-staining with FDA and PI. A mixture of FDA (final concentration $1.2 \mu \mathrm{M}$ ) and PI (final concentration of $2.5 \mu \mathrm{g} / \mathrm{mL}$ ) was added to cellular microtissues located in hMCA. The objects were simultaneously measured at the two wavelengths. Additionally, FDA staining was used for the functional examination of the multicellular microtissues as a kinetic test for short-time measurements. The rate of FDA hydrolysis by intracellular non-specific esterase was measured in individual cellular microtissues and calculated from repeated periodic measurements (10 times, at 2 min intervals) and presented as linear slope.

Mitochondrial membrane potential was measured by TMRM staining (final concentration $12.5 \mathrm{nM}$ ) in complete medium at $37^{\circ} \mathrm{C}$ under humidified atmosphere with $5 \% \mathrm{CO}_{2}$ for $1 \mathrm{~h}$. Surface marker expression was evaluated by direct immunofluorescent staining. A modified protocol was developed for staining of living 3D objects embedded in agarose in situ. Upon completion of vital measurements, the entire medium was removed from the macrowell, microtissues were washed twice with 0.5\% BSA in PBS at least $1 \mathrm{~h}$ per washing $\left(4^{\circ} \mathrm{C}\right)$ and thereafter, direct-stained by appropriate $\mathrm{mAbs}(1: 10)$ overnight at $4^{\circ} \mathrm{C}$.

For histological examination, the staining by hematoxylin-eosin (H\&E) dyes was performed as end-point procedures. Previously, multicellular breast cancer microtissues were fixed in situ by formalin and embedded in paraffin, with agarose "sandwich" intact. Paraffin sections of $5 \mathrm{~mm}$ thickness were prepared, deparaffinized and rehydrated. Regular protocol for H\&E staining was followed in the conventional manner.

\section{Imaging system and operating software}

Images were acquired using a motorized Olympus inverted IX81 microscope (Tokyo, Japan). The microscope was equipped with a sub-micron Marzhauser-Wetzlar motorized stage type SCANIM, with an Lstep controller; (Wetzlar-Steindorf, Germany) and a filter wheel including fluorescence filter cubes suitable for specific dyes (Supplementary Data Table S1). All filters were obtained from Chroma Technology Corporation (Brattleboro, VT). A 14-bit cooled, 
highly sensitive ORCA II C4742-98 camera (Hamamatsu, Japan) was used for imaging. A majority of the images were taken with a $\times 10$ magnification objective. The complete microscope system was enclosed in an incubator which provided a temperature of $37^{\circ} \mathrm{C}$ and humidified atmosphere containing $5 \% \mathrm{CO}_{2}$ (Life Imaging Services, Switzerland), allowing monitoring over long periods.

Image analysis: Olympus Cell^ $\mathrm{R}$ software (Tokyo, Japan) was used for image analysis. For optical data acquisition and analysis, each set of image acquisitions was initiated by acquiring the bright field (BF) image of a chosen view field, followed by the acquisition of several fluorescent

Images, one for each fluorescent probe, taken at a different preset time point. A series of regions on the hMCA was chosen and saved in a list of positions on the motorized stage. The initial cell distribution in each MC in those regions was imaged. For continuous monitoring of the microtissue formation process, the imaging system was programmed to take images of each saved position automatically, with consistent time intervals between each image, and the 6-well plate was either left on the microscope stage or placed in an external incubator and then returned to the microscope, verifying that the same regions were scanned, and images of the same spheroids taken.

Individual spheroids/microtissues were defined as regions of interest (ROI), and their sectional area (SA) was outlined on the BF image. Morphometric parameters of spheroids were extracted by image-processing algorithms of BF microscopy. Then, on each fluorescent wide field image, ROIs were determined by mapping those outlines on the interrogated fluorescent field image. Next, the fluorescent background, determined by averaging the fluorescence intensity (FI) detected by camera pixels found between the outlined regions, was subtracted from the fluorescent image. It should be emphasized that background signal determination and subtraction were performed separately for each of the acquired fluorescent field images. Then, fluorescent images were thresholded and mean FI value obtained for each ROI (all pixels within an object sectional area that are within the threshold borders) and the area percentage of these pixels (all fluorescent signals of a ROI) relative to the total sectional area of a ROI were calculated.

Statistical analysis: Each test was performed at least in duplicate (2 macro-wells), and 10-12 images from different areas within the hMCA were acquired, yielding images of about 120 individual MCs, and the corresponding spheroids per single macrowell, and about double for each test. The mean and SD for each measured parameter was calculated for the different spheroid populations under investigation. Comparisons between groups were performed using the t-test with statistical significance of differences at $\mathrm{P}<0.05$ for groups with Gaussian distributions and ANOVA for assessment of more than 2 groups. K-S test was used as non-parametric and distribution free criterion to non-normal data evaluation. Additionally, a machine learning linear algorithm (SVM) was used for analysis of microtissue growth rate as a function of initial number of cells generating growth under the various stiffness conditions.

\section{Results}

\section{Mechanical properties of hydrogels}

Hydrogels were made by dissolution of agarose powder (w/v) in PBS (Supplementary Data Table S2). The mechanical response of agarose hydrogels ( $\mathrm{G}^{*}$ complex modulus) borders on the limits [10] for pathological breast tissue. The measured complex modulus values of $1 \% \mathrm{w} / \mathrm{v}$ and $2 \% \mathrm{w} / \mathrm{v}$ agarose hydrogels are close to those measured by others for breast cancer tissue $(1.7 \mathrm{kPa}$ and $3.6 \mathrm{kPa}$, respectively), while those of higher concentrations of agarose rose significantly, approaching the upper limit and did not differ between $3 \% \mathrm{w} / \mathrm{v}$ and $6 \% \mathrm{w} / \mathrm{v}$ (about $10.5 \mathrm{kPa}$ ).

\section{Cellular spheroid formation and growth in hMCA}

Video 1: 


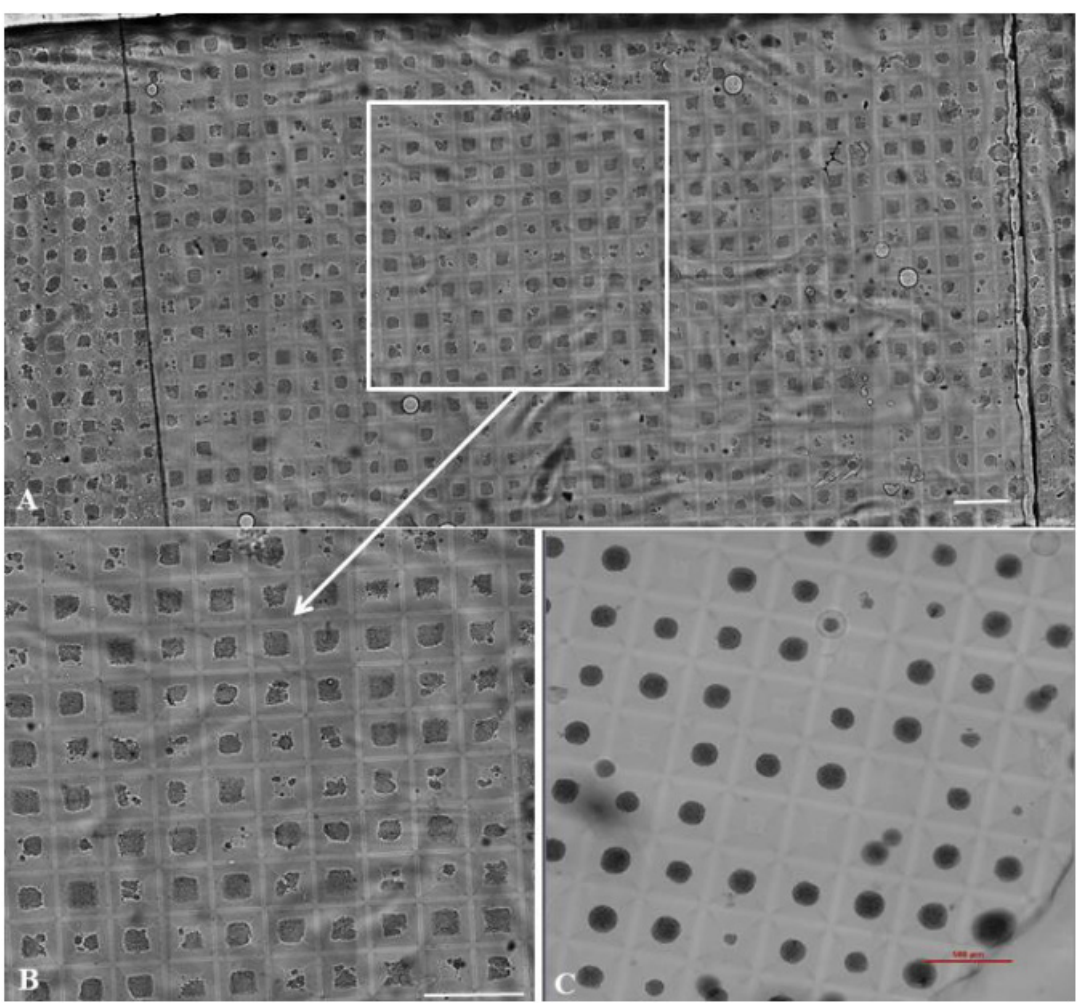

Arrayed format of the MCF-7 3D objects grown in the condition of $1.7 \mathrm{kPa}$ introduced directly after cell seeding into hMCA is shown. Wide bright field stitch image from the single macro-well consists 720 three-dimensional objects $(\mathrm{A})$ is accompanied by the enlarged representative area indicated by rectangle in the stitch image for illustration (B). Representative area of the control 3D spheroids grown in the regular condition of low rigidity (about 400 objects per macro well) is presented (C). Magnification $\times 10$. Scale bar $0.5 \mathrm{~mm}$.

Figure 1: MCF-7 3D objects grown by arrayed format within surroundings of different rigidity for seven days.

MCF-7 human breast cancer cell capability to develop in vitro by arrayed format, as described in Methods (Figure 1) and in 3D microtissue is performed under different rigidity conditions Supplementary Material (Video S3).

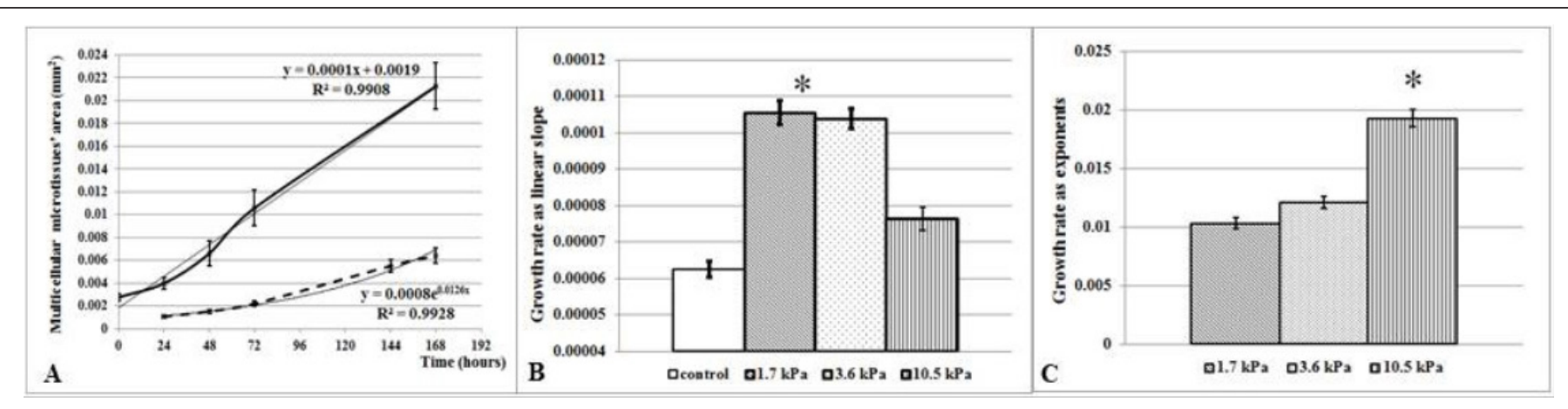

MCF-7 cells were loaded in the agarose chip and grown as 3D objects within the agarose layer by different stiffness for seven days. (A) Growth curves of MCF-7 microtissue population is presented as increase of microtissue SA (mm2) (ordinate) via time (hours) of growth (abscissa). First point of each graph presents SA of microtissues grown $24 \mathrm{~h}$ after cell seeding either freely (smooth lines) or within agarose layer (dashed line). The increase in SA of microtissues within stiffer surrounding (3.6 kPa) is presented as mean $\pm \mathrm{SD}$ at each time point and accompanied by trendline for each graph. Growth rate was calculated as linear slope (B) of the microtissue SA increase in $19 \mathrm{~h}$ embedded microtissues or as exponents (C) in $0 \mathrm{~h}$ embedded objects. Asterisks indicate the significant differences calculated as t-test in the groups of microtissues grown from cells embedded within agarose overnight after the seeding; or as ANOVA in the groups of microtissues grown from cells embedded within agarose immediately after seeding.

Figure 2: Multicellular 3D object growth is dependent on the initial mechanical conditions. 
Examination of MCF-7 microtissue growth revealed the different types of microtissue SA increases, dependent on the point in time of agarose embedding. When initial cellular conglomerates created by cells during the first $19 \mathrm{~h}$ of incubation in the hMCA MCs (Figure 2A) were embedded in the agarose, the increase in microtissue SA had a typically linear character, allowing evaluation of microtissue growth rate using a linear slope (Figure 2B). The linear character of the 3D object growth is in agreement with previously published studies [27] and similar to control spheroids grown under regular conditions. When cells were embedded by agarose at $19 \mathrm{~h}$ after seeding, microtissue growth rate was significantly greater in the groups of microtissues grown under conditions of $1.7 \mathrm{kPa}$ or 3.6 $\mathrm{kPa}$ stiffness, in comparison with control freely grown spheroids $(\mathrm{P}<0.000001)$. The growth rate of microtissues grown under 10 $\mathrm{kPa}$ stiffness from $19 \mathrm{~h}$ after seeding slowed in comparison to those grown in more relaxed stiffness conditions $(\mathrm{P}<0.00001)$, nevertheless exceeding that of control samples $(\mathrm{P}<0.0003)$.

In contrast, when MCF-7 cells were embedded in agarose immediately after loading into hMCA, before early multicellular conglomerates were established, a delay of about $36 \mathrm{~h}$ was discovered in the microtissue SA increase, and an exponential character of the microtissue growth was observed independently from extent of stiffness (Figure 2A). However, the growth rate calculated as exponent (Figure 2C) for each individual 3D object is greater for 3D objects grown in stiffer conditions $(\mathrm{P}<0.00001)$.
Hence, in vitro microenvironmental rigidity can affect the growth rate of 3D tumor microtissues at least during the first seven days of culturing. Increased rigidity introduced both on the early conglomerates and on single cells loaded directly, promotes microtissue development in comparison to more relaxed rigidities.

\section{Association between MCF-7 spheroid growth and number of cells seeded}

Analysis of average microtissue growth rate under the different rigidities was complemented with a detailed analysis of microtissue growth rate of 3D objects initiated from the same number of cells under various rigidity conditions of (Figure 3). A count of the number of cells revealed that $96 \%$ of all 3D objects were initiated from 1 to 33 cells loaded per individual MC when cells were embedded into agarose at $19 \mathrm{~h}$ after loading. No correlation was found between number of cells initiating the microtissue growth and the corresponding growth rate in entire populations of the 3D microtissue under each stiffness condition (Figure 3A). However, surprisingly, a significant difference (Figure 3C) in growth rate was found in the group of $3 \mathrm{D}$ objects which were initiated by one to eight cells $(\mathrm{P}<0.006$ in $1.7 \mathrm{kPa}$ stiffness, $\mathrm{P}<0.02$ in $3.6 \mathrm{kPa}$ stiffness, $\mathrm{P}<0.0001$ in $10.5 \mathrm{kPa}$ stiffness and $\mathrm{P}<0.004$ in control freely grown spheroids). When 3D objects were initiated by more than 15 cells, no significant difference in growth rate was revealed under each stiffness condition.

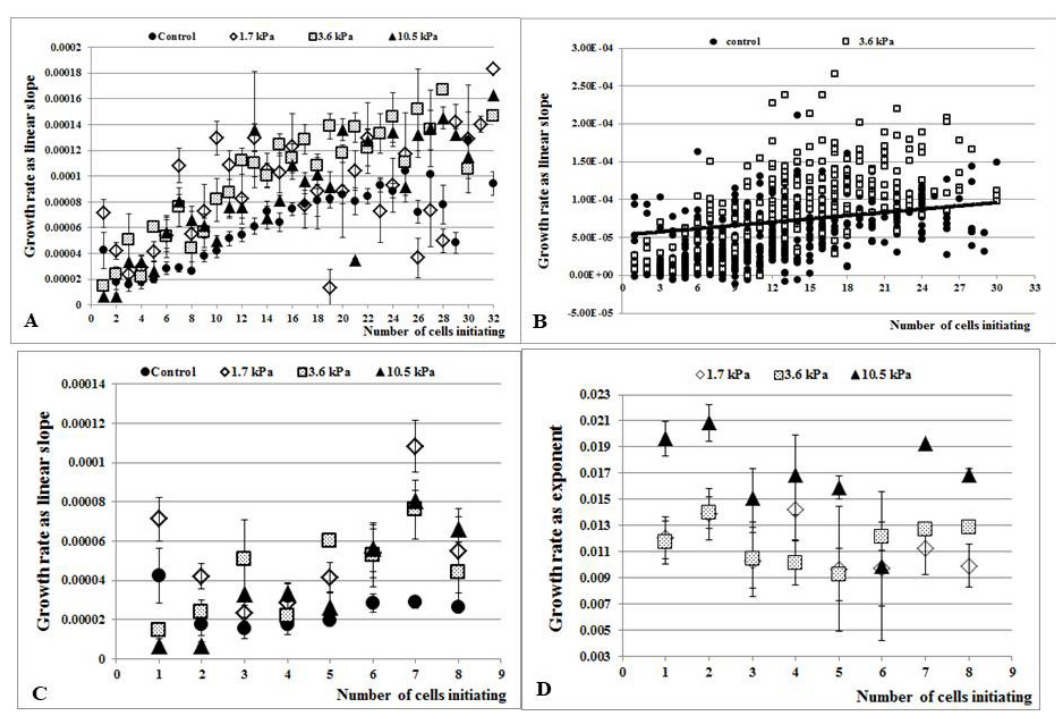

Microtissues were grown and measured as described in Materials and Methods. Each dot is the average growth rate (mean \pm SE) calculated in the groups with equivalent initial number of cells. Growth rate (ordinate) vs number of cells seeded (abscissa) is presented. (A) Growth rate was calculated as a linear slope of SA increase for each individual microtissue grown from cells embedded in agarose $19 \mathrm{~h}$ after seeding (B) The linear classifier calculated by SVM algorithm distinguishes between control spheroids and microtissues grown under 3.6 $\mathrm{kPa}$ rigidity depending on the initial number of cells generating growth. (C) Enlarged part of the graph (A) presents the growth rate of groups of microtissues initiated by one to eight cells.

(D) Growth rate was calculated as exponent of the SA increase for each individual microtissue grown from cells embedded in agarose immediately after seeding.

Figure 3: Association between 3D object growth rate and number of MCF-7 cells initiating their creation and growth 
Additional analysis of 3D object growth rate considering the initial number of cells was performed by calculating the linear classifier using an SVM algorithm to distinguish between control, freely grown spheroids and 3D microtissues grown under stiffer conditions. The calculated line (Figure 3B)

\section{$-1.8186 \times 104$ (slope) +0.0261 (number of cell) $+0.97=0$}

Successfully separates microtissues grown under stiffer conditions from control, freely grown spheroids with an accuracy of $77 \%$ ( $23 \%$ misclassified cases for each group), even in relatively small data samples (for example, 674 and 248 samples in control data and experimental 3D objects grown in $3.6 \mathrm{kPa}$ condition, respectively).

In contrast, growth rate had been found to be independent of the initial number of cells in the microtissues initiated by cells embedded in agarose immediately after seeding (cell count reveals 1-21 cells per MC). In other words, detected variances in microtissue growth rate in different rigidity (see above) are not associated directly with initial amount of cells seeded in individual MCs (Figure 3D). It is indeed observed that average growth rate of the microtissues grown under $10.5 \mathrm{kPa}$ stiffness is higher than that of microtissues grown in softer agarose layers. However, under each stiffness condition, growth rate exhibited no difference $(\mathrm{P}=0.6$ in $1.7 \mathrm{kPa}$ and $3.6 \mathrm{kPa}$ stiffness, $\mathrm{P}=0.2$ in $10.5 \mathrm{kPa}$ stiffness, respectively).

Hence, since analysis for each individual 3D object began with the number of cells initiating its creation, the variability created by the number of cells per individual MC can be avoided, suggesting that stiffer microenvironment may be a significant factor in $3 \mathrm{D}$ breast cancer microtissue growth in vitro, especially when the 3D objects are initiated only by few individual cells. Said result supports the hypothesis that growth of 3D objects depends on the rigidity of the environment and the point in time at which stiffness is induced.

\section{Morphometric analysis of breast cancer spheroids grown under conditions of different rigidity}

Seven-day 3D breast cancer microtissues demonstrated morphology variability even when grown under the same experimental conditions (Figure 4). We evaluated morphology variances of the multicellular 3D objects developed in microenvironments of varying rigidity, scored at end point after seven days of growth.

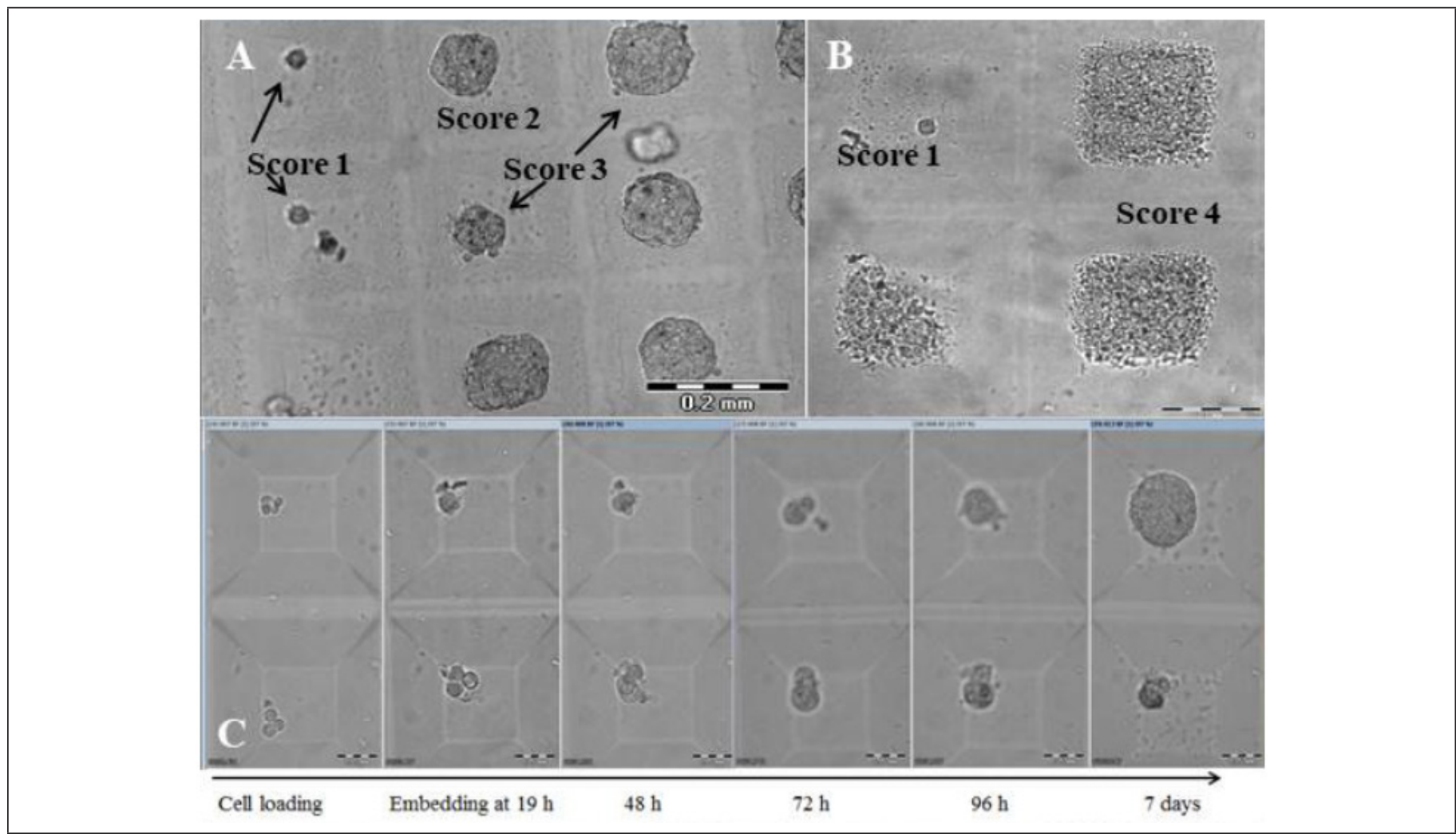

Seven-day MCF-7 microtissues demonstrate different morphology according to 3D object surface smoothness, cell arrangement and edge texture. (A) Three of four different morphological types are presented (see text below). Scale bar $0.2 \mathrm{~mm}$. (B) A single 3D object (Score 1) and three Score 4 microtissues are presented. Scale bar $0.1 \mathrm{~mm}$. (C) Representative sample of MCF-7 microtissues grown in stiffer (1.7 $\mathrm{kPa}$ ) environment demonstrates the seven-day growth of MCF-7 microtissues of Score 3 (upper panel) and Score 1 (lower panel) which were initiated by four cells each embedded in agarose overnight after seeding. Scale bar $0.05 \mathrm{~mm}$.

Figure 4(A,B,C): Morphological groups and histological structure of seven-day MCF-7 3D microtissues. 
Morphological score included assessment of spheroid surface texture, smooth or uneven; cell arrangement; and edge quality, smooth or rough. Four groups of spheroids were classified accordingly:

A. rough configuration;

B. round morphology with smooth edges;

C. round morphology with smooth edges and single peripheral cells; and

D. loose morphology with unclear edges and massive peripheral cell spread

A significant shift in favor of the spheroids with unclear edges and massive peripheral cell spread (Score 4) was observed in those spheroids embedded in agarose. More than 30 percent of spheroids embedded in agarose at time $19 \mathrm{~h}$ after cell seeding, demonstrated unclear (ambiguous) edges, while no such 3D objects were observed in control freely grown spheroids (Table 1). When seeded cells were embedded in agarose immediately after sedimentation, this shift was even more pronounced. At least half of the 3D objects grown under more rigid surroundings exhibit loose morphology $(\mathrm{P}<0.01)$. Moreover, loose morphology 3D objects also demonstrated dispersed intrinsic structure (Figure 4C \& 4D) in comparison with compact cellular structure of 3D objects in the groups with round morphology (Scores 2 and 3). The randomness and SD of the gray values in the images were assessed for evaluation of the intrinsic texture in 3D object SA, and revealed significant increases in both parameters of Score 4 objects as compared to the more compact Score 2 and 3 microtissues $(1.6 \pm 0.01$ vs $1.4 \pm 0.02$ in gray value entropy, $\mathrm{P}<0.00001$; and $230.5 \pm 2.5$ vs $189.9 \pm 3.5$ in gray value $\mathrm{SD}, \mathrm{P}<0.00001)$.
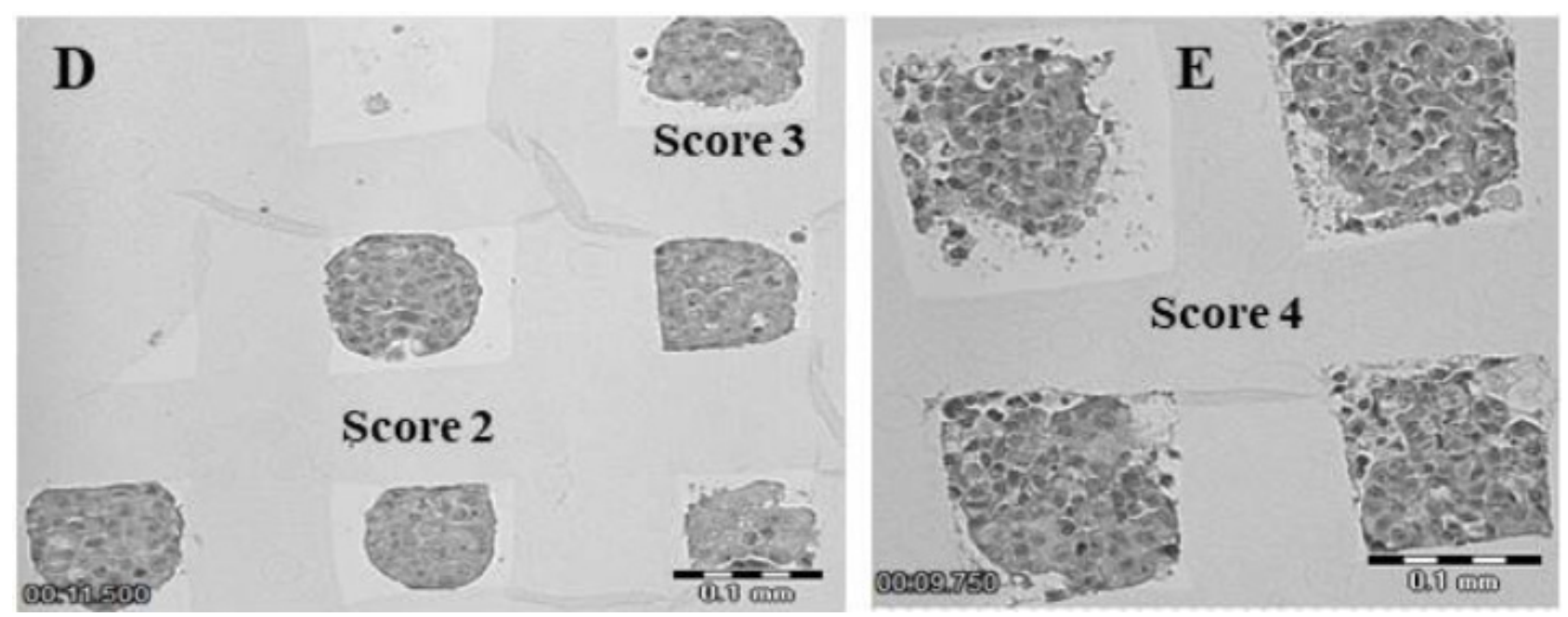

Seven-day MCF-7 microtissues demonstrate different morphology according to 3D object surface smoothness, cell arrangement and edge texture. $(D, E)$ Histological examination demonstrated different intrinsic structure of the seven-day MCF-7 microtissues grown in stiffer condition (10.5 kPa): (E) Score 4 group microtissues possess a more dispersed intrinsic structure in comparison to Score 2 and 3 group spheroids (D). Magnification $\times 10$. Scale bar $0.1 \mathrm{~mm}$.

Figure 4(D,E): Morphological groups and histological structure of seven-day MCF-7 3D microtissues.

\begin{tabular}{|c|c|c|c|c|}
\hline \multirow{2}{*}{ Table 1: Morphology variants among MCF-7 microtissues grown under conditions of different rigidity. } \\
\hline \multirow{2}{*}{ Agarose stiffness } & \multicolumn{4}{|c|}{ Embedding at 0 h after seeding } \\
\cline { 2 - 5 } & Score 1 (\%) & Score 2 (\%) & Score 3 (\%) & Score 4 (\%) \\
\hline $1.7 \mathrm{kPa}$ & $18.9 \pm 5.5$ & $36.8 \pm 6.1$ & $33.6 \pm 11.5$ & $10 \pm 13.8$ \\
\hline $3.6 \mathrm{kPa}$ & $24.1 \pm 18.5$ & $12 \pm 17$ & $10 \pm 12$ & $53.2 \pm 32$ \\
\hline $10.5 \mathrm{kPa}$ & $15.8 \pm 13.2$ & $12.9 \pm 22.7$ & 0.001 & 0.005 \\
\hline $\mathrm{P}$ & 0.3 & 0.004 & Score 3 (\%) & Score 4 (\%) \\
\hline \multirow{2}{*}{ Agarose stiffness } & & Embedding at 19 h after seeding & 29.9 \\
\hline $1.7 \mathrm{kPa}$ & Score 1 (\%) & $20.7 \pm 13.7$ & $30.2 \pm 21.9$ & 29.9 \\
\hline $3.6 \mathrm{kPa}$ & $19.2 \pm 13.4$ & $10.4 \pm 8.2$ & $47.8 \pm 24.5$ & $29.8 \pm 22.7$ \\
\hline $10.5 \mathrm{kPa}$ & $11.2 \pm 9$ & $13.9 \pm 7.2$ & $25.4 \pm 14.1$ & $39.2 \pm 31.1$ \\
\hline
\end{tabular}




\begin{tabular}{|c|c|c|c|c|}
\hline Control & $16.3 \pm 12.4$ & $36.8 \pm 12.9$ & 0 & $46.9 \pm 16.3$ \\
\hline $\mathrm{P}$ & $0.9 / 0.8$ & $0.0002 / 0.3$ & $0.06 / 0.3$ \\
\hline
\end{tabular}

$P$ was calculated as ANOVA and represents a comparison within each score group of the various rigidities in the microtissues grown from cells embedded in agarose at $0 \mathrm{~h}$ (upper table) and $19 \mathrm{~h}$ (lower table) after seeding. In the latter case, the number to the left of the slash represents a comparison between percentages of scored microtissues in all rigidities including control low rigidity, while the number to the right of slash between percentages of scored microtissues grown under conditions of higher rigidities only, excluding control.

When microtissue growth rate was analyzed within each morphology group separately, the match of this parameter was revealed for microtissues embedded in various agarose stiffnesses at time $19 \mathrm{~h}$, i.e. spheroids with the same morphology demonstrated similar growth rate independently of their surrounding stiffness $(\mathrm{P}=0.09)$. In contrast, when cells were embedded in agarose immediately after seeding in the hMCA, growth rate of the microtissues grown in stiffer environments differs significantly in the same morphology group. In other words, Score 3 and 4 spheroids grew faster in stiffer milieu (Table 2). Score 1 microtissues were shown to grow similarly in all stiffness conditions $(\mathrm{P}=0.4)$.

Table 2: Growth rate of the different morphology variants of microtissues embedded in agarose immediately after cell seeding.

\begin{tabular}{|c|c|c|c|c|}
\hline Agarose stiffness & $\mathbf{1 . 7} \mathbf{~ k P a}$ & $\mathbf{3 . 6} \mathbf{k P a}$ & $\mathbf{1 0 . 5} \mathbf{~ P a}$ & $\mathbf{P}$ \\
\hline Score 1 & $0.005 \pm 0.007$ & $0.006 \pm 0.005$ & $0.007 \pm 0.0009$ & 0.4 \\
\hline Score 2 & $0.0097 \pm 0.006$ & $0.013 \pm 0.004$ & $\mathrm{NA}$ & 0.07 \\
\hline Score 3 & $0.0098 \pm 0.005$ & $0.014 \pm 0.005$ & $0.022 \pm 0.008$ & 0.0014 \\
\hline Score 4 & $0.009 \pm 0.005$ & $0.015 \pm 0.0006$ & $0.02 \pm 0.006$ & $4.2 \times 10^{-5}$ \\
\hline P* $^{*}$ & $1.5 \times 10-7 / 0.9$ & $8.3 \times 10-7 / 0.3$ & $1.5 \times 10-5 / 0.9$ & \\
\hline
\end{tabular}

MCF-7 cells were loaded in hMCA and embedded by agarose immediately after seeding as described in Materials and Methods. Morphology variances of the 3D multicellular microtissues were scored at end point after seven days growth. Growth rate was calculated as exponents for each individual 3D object. $\mathrm{P} / \mathrm{P}^{*}$ were calculated as ANOVA. $\mathrm{P}$ represents comparison within each score group of the various rigidities. $\mathrm{P}^{*}$ represents comparison of rigidities between score groups: number to the left of slash - between rigidities of all score groups and number to the right of slash - between rigidities of Score groups 2, 3 and 4.

Additionally, no correlation was revealed between morphology variants and initial number of cells seeded (Figure 4C). MCF-7 cells are a strong epithelial phenotype with Ep-CAMhigh expression which is needed as a growth- and invasion-promoting factor [28]. CXCR4 protein expression on breast cancer cells has been found to be significantly associated with lymph node metastasis and TNM stage in clinical studies [29]. Seven-day 3D microtissues were direct double-stained by anti-Ep-CAM and anti-CXCR4 Abs and expression rate in the area which demonstrated strong fluorescent intensity, was assessed as a percentage of the whole ROI.
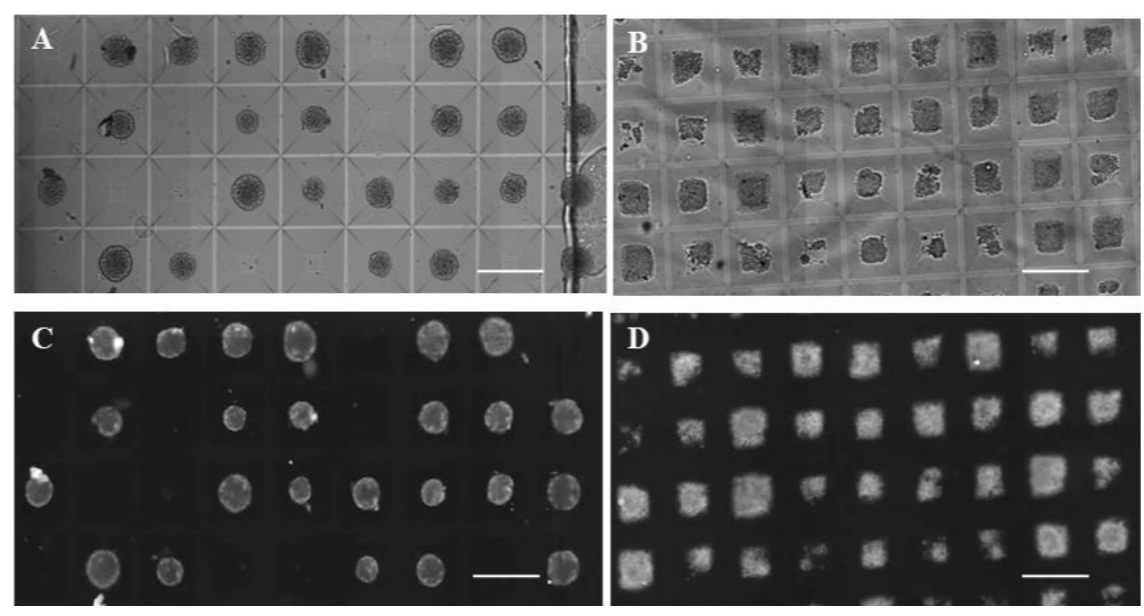

MCF-7 3D objects were grown and stained as described in Materials and Methods. Bright field $(A, B)$ and corresponding fluorescent EpCAM-FITC images $(C, D)$ of the control MCF-7 spheroids grown under regular low rigidity conditions $(A, C)$ and $3 D$ microtissues grown under conditions of higher rigidity $(1.7 \mathrm{kPa})$ are presented $(B, D)$. Magnification $\times 10$. Scale bar $0.25 \mathrm{~mm}$.

Figure 5: Ep-CAM expression on MCF-7 microtissue surface. 
A significant decrease in Ep-CAM expression was revealed when 3D objects were grown under stiffer conditions as compared to free floating grown spheroids $(\mathrm{P}<0.0002)$. About $20 \%$ decrease of the stained area was observed in the 3D microtissues grown under $3.6 \mathrm{kPa}$ rigidity (Figure 5), while no difference was observed in expression levels of the surface CXCR4 chemokine in the 3D objects grown under stiffer conditions in comparison to freely grown spheroids $(\mathrm{P}=0.17)$. Additionally, the point in time at which seeded cells were embedded with agarose did not influence surface Ep-CAM/CXCR4 expression.

\section{Vital parameters of MCF-7 microtissues grown in different stiffness surroundings}

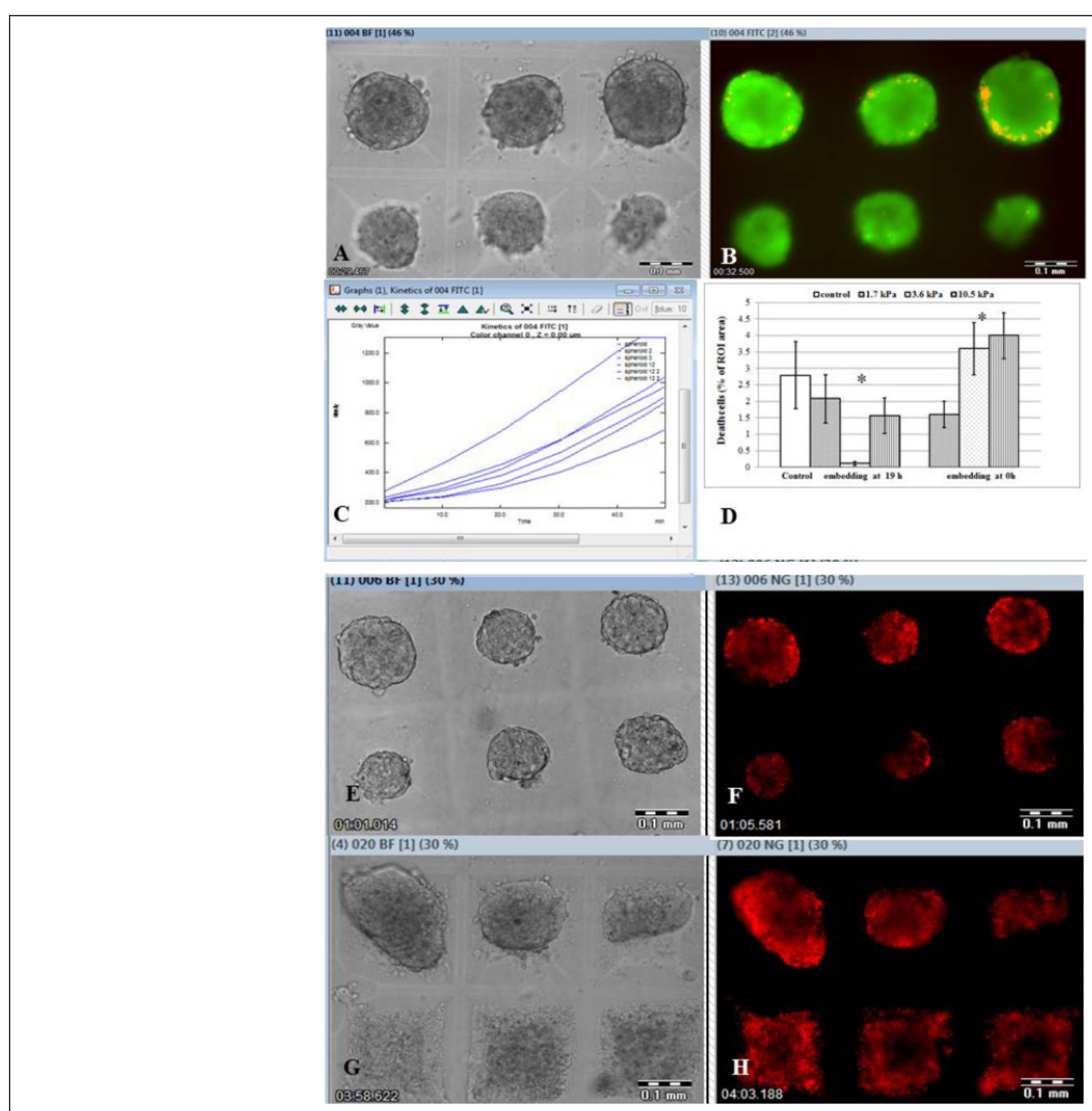

MCF-7 3D objects were grown and stained as described in Materials. Bright field $(A, E, G)$ and corresponding fluorescent images (B,F,H) of the MCF-7 3D microtissues grown under conditions of higher rigidity are presented. Magnification $\times 10$. Scale bar $0.1 \mathrm{~mm}$.

Seven-day microtissues grown from the MCF-7 cells embedded under $1.7 \mathrm{kPa}$ immediately after seeding (A) were double stained by FDA (green staining) and PI (red staining) in situ. Corresponding merged images of FDA staining indicate live cells, while red (PI) indicate dead cells which are rarely seen (B). Representative sample of FDA hydrolysis within MCF-7 seven day microtissues embedded within $3.6 \mathrm{kPa}$ stiffness at $19 \mathrm{~h}$ is demonstrated as increase of the intracellular fluorescein FI (C). PI positive area within microtissue SA determines the dead cells and is presented in the graph where asterisks demonstrate the statistically significant difference between experimental and control populations (D). Representative samples of the 3D microtissues grown under $1.7 \mathrm{kPa}$ introduced directly after cell seeding (E,F) or overnight after cell seeding (3.6 kPa) into hMCA $(\mathrm{G}, \mathrm{H})$ and stained by TMRM in situ are shown at wide bright field image $(\mathrm{E}, \mathrm{G})$ accompanied by corresponding fluorescent images $(\mathrm{F}, \mathrm{H})$.

Figure 6: Vital staining of MCF-7 microtissues grown in different stiffness surroundings.

Semi-quantitative morphology score and surface marker expression assessment were completed by phenotypic estimation of vital parameters of the $3 \mathrm{D}$ objects using three fluorescent parameters measured: PI positive area within SA for the 
determination of dead cells, intracellular FDA hydrolysis for the assessment of cellular metabolic activity and TMRM fluorescent intensity for the estimation of mitochondrial membrane potential (Figure 6) as described in Methods.

Very low autofluorescence of agarose hydrogel at green and red wavelengths significantly improves low-intensity signal detection (Figure 6) due to much higher cellular signal vs. well wall ratio. The PI positive area within SA, indicating the dead cells, was calculated as a percentage of the whole SA area. Despite an overall small PI positive area (Table 3), the control spheroids which were grown freely demonstrated significantly larger PI positive areas in comparison to microtissues that were embedded in agarose at 19 $\mathrm{h}$ after cell seeding $(\mathrm{P}<0.01)$. The smallest $\mathrm{PI}$ positive area is seen in microtissues grown in $3.6 \mathrm{kPa}$ stiffness compared to samples embedded in agarose with other degrees of stiffness $(\mathrm{P}<0.008)$. When microtissues grown in agarose from time $0 \mathrm{~h}$ (embedding immediately after cell seeding), the stiffer environment caused a significant increase in the size of the PI positive area $(3.6 \pm 0.8 \%$ under $3.6 \mathrm{kPa}$ and $4 \pm 0.7 \%$ under $10.5 \mathrm{kPa}$ ) compared to softer ones $(\mathrm{P}<0.01)$, including control samples grown without agarose embedding.

Table 3: Vital parameters in microtissues grown from cells embedded at $19 \mathrm{~h}$ after seeding.

\begin{tabular}{|c|c|c|c|c|c|}
\hline \multirow{2}{*}{ Agarose stiffness } & \multicolumn{4}{|c|}{ FDA hydrolysis as FI linear slope } & \multirow{2}{*}{ PI (\% of SA) } \\
\cline { 2 - 6 } & Score 1 & Score 2 & Score 3 & Score 4 & $2.1 \pm 0.6$ \\
\hline $1.7 \mathrm{kPa}$ & $14.6 \pm 14$ & $7.3 \pm 6.7$ & $6.6 \pm 9$ & $7.7 \pm 11.5$ & $0.1 \pm 0.01 *$ \\
\hline $3.6 \mathrm{kPa}$ & $5.1 \pm 1.2$ & $7.5 \pm 3.4$ & $17.5 \pm 3.8$ & $12.2 \pm 2.9$ & $1.6 \pm 0.5$ \\
\hline $10.5 \mathrm{kPa}$ & $8.5 \pm 3.2$ & $12.4 \pm 3.7$ & $2.6 \pm 2.1$ & $\mathrm{NA}$ & $2.8 \pm 1$ \\
\hline Control & $3.5 \pm 4.2$ & $6.7 \pm 13.4$ & $1.9 \times 10-5$ & $3.1 \times 10-5$ & \\
\hline $\mathrm{P}$ & 0.3 & 0.07 & & \\
\hline
\end{tabular}

Seven-day microtissues were grown and double stained by FDA and PI in situ. The FDA hydrolysis rate and PI positive area were calculated as described in Materials and Methods. P was calculated as ANOVA in score groups; ${ }^{*}$ indicates the difference in PI positive areas in microtissues grown under the stiffer conditions.

Weak PI staining showed rare cellular death events accompanied by strong FDA staining which is a known indicator of intracellular metabolic activity (Supplementary Data Video S4). No microtissues were detected negative according to FDA staining - neither in experimental, nor in control objects. The rate of FI increase is significantly slower in spheroids grown freely in comparison with those objects grown within stiffer milieu $(\mathrm{P}<3.4 \times 10-7)$ when cells were embedded overnight after seeding (Figure 6C \& 6D). Moreover, when FDA hydrolysis ability was analyzed in the various morphological groups, Score groups 3 and 4 3D objects grown in the stiffest surroundings $(10.5 \mathrm{kPa})$ demonstrated better ability to hydrolyze FDA, as opposed to those grown in softer environments (Table 3).

\section{Video 2:}

Surprisingly, averaged TMRM FI is independent of both the environmental rigidity $(\mathrm{P}<0.7)$ and the point in time at which embedding was performed $(\mathrm{P}<0.1)$. However, when the morphology score is taken into account, a trend to lower mean FI in the Score 1 group is observed for each rigidity, while the other score groups remain similar in this parameter.

\section{Discussion}

Breast cancer development is characterized by the combined activity of the epithelium, tumor-associated vasculature, and microenvironmental cellular and extracellular components, resulting step-by-step, in the transition from normal state to invasive carcinoma. A mutual interaction exists between cancer cells and their microenvironment, invariably causing loss of normal tissue integrity and cancer cell spread. One of the important elements in this process is changing tissue rigidity which exhibits incremental stiffening [30], regulates tumor-microenvironment crosstalk and supports tumor progression and expansion. The pronounced impact of surrounding rigidity on multicellular 3D breast cancer model formation and development in vitro was demonstrated in this study. Alterations in growth rate, external and intrinsic structures, as well as in expression of epithelial differentiation molecules under stiffer conditions denote the crucial need to better simulate in vitro, the mechanical conditions of tumor development in vivo.

We present an effective approach to modify the surrounding rigidity in vitro, in order to mimic the mechanical properties within primary tumor tissues and during cell invasion and expansion. Most 
current protocols preserve constant surroundings during formation and growth of multicellular 3D structures [31,32] in general, and in particular, during formation of 3D breast cancer spheroids $[33,34]$. As a rule, additives such as methocel, collagen or Matrigel, used for improving 3D spheroid formation, do not notably alter surrounding stiffness. For example, rigidity of collagen used for 3D breast cancer spheroid formation does not exceed the stiffness of normal breast tissue $[7,10]$. In other words, in vitro multicellular 3D breast cancer structures are developed under mechanical conditions similar to those in normal breast tissue, yet unlike the actual status in vivo.

The stiffer conditions used in this study are associated with the appearance of morphologically diverse 3D microtissue subgroups, which demonstrate different functional abilities with respect to enzymatic and mitochondrial activity. This surprising discovery can be correlated with the histological structure of primary invasive breast tumor tissue which exhibits significant intratumoral heterogeneity by morphological patterns of cancer cells $[35,36]$. Different morphologic type (alveolar, solid, tubular, trabecular and discrete) structures have been shown to form from transcriptionally distinct subpopulations of tumor cells $[37,38]$. The capacity of dissociated mice tumor cells to reconstruct their original histological structure in vitro was demonstrated previously (1959) and the exhibited histological pattern bore a basic resemblance to that of primary tumor tissue [39]. Based on this information, the Score 2 (round regular morphology with smooth edges) 3D microtissues and possibly the Score 3 (round morphology with smooth edges and single peripheral cells) 3D microtissues as well, can be interpreted as similar to alveolar structures, while the Score 4 (with "pre-invasive" phenotype) 3D microtissues better mimic solid structures with looser histology. Perhaps the Score 1 microtissues structurally resemble discrete structures arranged by either a few cells or a single cell. So, a more rigid environment in vitro contributes to 3D microtissue growth and its structural and functional diversity resembling actual structures in the body. Additionally, it is now considered that MCF-7 cells have a low metastatic potential. However, a decrease of surface Ep-CAM expression in the 3D objects grown under stiffer conditions may suggest the switch between the strong epithelial phenotype and the less differentiated cellular phenotype [40], and together with the unaltered expression of surface CXCR4 chemokine, facilitate metastatic potential.

Secondly, the point in time at which agarose embedding is performed is a second determinative factor. Embedding by agarose, of cells loaded at time zero may reproduce invasive conditions when tumor cells invade an already existing microenvironment of higher rigidity, while embedding of early cellular conglomerates (19 h after cell seeding) may imitate the initial step of tumor development when tumor cells start to proliferate and modify ECM rigidity to become stiffer. The first protocol (embedding immediately after cell loading), aside from stiffness, closely resembles major current protocols [32], while the latter is quite different in that cells begin to form multicellular objects under lower stiffness conditions and continue to proliferate while surrounding rigidity changes in situ. This unique method enables altering physical properties of the 3D model culturing, without the necessity of transferring each conglomerate elsewhere (wells, plate), unlike methods described in literature [31] and optionally, with or without simultaneous biochemical influences.

Thirdly, the number of initiating cells is the additional issue which plays a significant role in the ability of breast cancer cells to develop 3D structures in vitro. A majority of published studies have demonstrated spheroid formation from a relatively large number of cells (hundreds/thousands), sometimes specifying a minimum number of cells needed for generation of 3D objects either from the cell line [15,31,41] or from primary mammary cells [14]. As a general rule, the number of cells for $3 \mathrm{D}$ spheroid formation is presented at concentration units, assuming a homogenous distribution of cells per device/macro-well. The easy control of spheroid size and growth rate by initial number of seeded cells is described and discussed in our previous study [42] as well. However, the primary tumor is initiated by individual cells during accumulation of somatic mutations resulting in uncontrollable cell proliferation and tumor tissue growth. So, analysis of 3D microtissues initiated by single cells (clone-forming 3D object), as opposed to multicellular 3D objects initiated by groups of cells, has particular importance for understanding primary tumor development, as well as for understanding individual cell invasion and metastasis. In this study we demonstrated the process of spheroid/microtissue formation from only few cells, with the lowest limit being one individual cell. Formation of tumor microtissue from individual cells is of special interest in light of the information about specific phenotypic subpopulations of cancer-initiating/stem cells and their role in tumor progression, metastasis and therapy resistance [43]. Finally, development of more efficient and physiologically relevant models in vitro for evaluation of cancer tumor behavior and arrangement closer mimicking the actual status in vivo requires several fundamental research capabilities:

a. it is paramount for successful 3D structure formation, to avoid cell adhesion to surface, either by mechanical influence or use of chemical substances, as the absence of such forced immobilization allows observation, monitoring and measurements in a wider range of physiologic conditions;

b. to create mechanical conditions which simulate those in body without or with introduction of a desired chemical signal/ component; and

c. to identify and study the heterogeneity of microtissue formation and functional capabilities within entire populations using multiple functional repeated kinetic measurements.

Presented results meet the above requirements and may contribute to understanding the variety of individual tumors and 
thus, to the potential individualization of anti-cancer therapy. Conclusions

Physical changes of cell surroundings without parallel changes in biochemical conditions may present a good opportunity to study cell response to only mechanical signal in vitro. Environmental stiffness affects growth and development of cancer 3D microtissue in vitro.

A stiffer environment better supports 3D microtissue growth in comparison to regular low rigidity conditions. The morphologic diversity of the 3D structures was revealed. Four groups, differing in their morphology, have been discovered, which exhibited the various vital features associated with the extent of environmental rigidity and the point in time at which embedding was performed. Moreover, the Score 4 morphology group demonstrates higher pre-invasive potential. Additionally, surface epithelial marker expression significantly decreased in the 3D microtissues grown under stiffer conditions independently from the point in time of agarose embedding.

3D objects derived from less than eight cells are of special interest due to significant alteration in their growth behavior which is independent of surrounding rigidity. A number of intriguing questions remain unanswered:

a) does the growth rate of the $3 \mathrm{D}$ object initiated by less than eight cells change after proliferation cycles, at which time the number of cells within mictrotissue has grown?

b) how does the quantity of 3D objects initiated by one individual cell, which survive under stiffness conditions that resemble tumor tissue, compare to those which survive under low rigidity conditions? and

c) does a difference exist between invasive capacity of the daughter cells originating from "clone-forming" 3D objects and those originating from the 3D microtissues initiated by more than one cell? - all issues subject to further study.

\section{Notes}

\section{Acknowledgments}

This study was endowed by the Bequest of Moshe-Shimon and Judith Weisbrodt.

\section{Compliance with Ethical Standards}

\section{Conflicts of Interest}

The authors declare that they have no financial conflicts of interest.

\section{Ethical Statement}

There have been no human or animal experiments carried out for this article.

\section{Author Contributions}

a. Elena Afrimzon initiated and led this study, carried out experiments, image analysis and assessment of the results. She performed the literature survey and prepared the manuscript.

b. Sergei Moshkov was responsible for design of the HMC array and its production.

c. Yana Shafran contributed to in-HMC generation and culturing of breast cancer spheroids.

d. Ronen Pelov led the rheometric measurements of the hydrogels. Maya Freund led the histological examination of breast cancer microtissues.

e. Yaron Hakuk was responsible for software programs and contributed to image analysis.

f. Zahavit Bar-On-Eizig was responsible for image analysis.

g. Naomi Zurgil supervised this study and drafted the manuscript.

h. Mordechai Deutsch supervised this study, designed and coordinated HMC array production and drafted the manuscript.

The manuscript has been read and approved by all named authors and there are no other persons who satisfy the criteria for authorship who are not listed.

\section{References}

1. Ho JC, Ueda J, Shimizu T (2016) The impact of mechanical stress on stem cell properties: The link between cell shape and pluripotency. Histol Histopathol 31(1): 41-50

2. Hallmann R, Zhang X, Di Russo J, Li L, Song J, et al. (2015) The regulation of immune cell trafficking by the extracellular matrix. Curr Opin Cell Biol 36: 54-61.

3. Adamo L, Naveiras O, Wenzel PL, McKinney-Freeman S, Mack PJ, et al. (2009) Biomechanical forces promote embryonic haematopoiesis. Nature 459(7250): 1131-1135.

4. Moraes C, Sun Y, Simmons CA (2011) (Micro)managing the mechanical microenvironment. Integr Biol (Camb) 3(10): 959-971.

5. Hao J, Zhang Y, Jing D, Shen Y, Tang G, et al. (2015) Mechanobiology of mesenchymal stem cells: Perspective into mechanical induction of MSC fate. Acta Biomater. 20: 1-9.

6. Kumar S, Weaver VM (2009) Mechanics, malignancy, and metastasis: The force journey of a tumor cell. Cancer Metastasis Rev 28(1-2): 113-127.

7. Paszek MJ, Zahir N, Johnson KR, Lakins JN, Rozenberg GI, et al. (2005) Tensional homeostasis and the malignant phenotype. Cancer Cell 8(3): 241-254.

8. Mueller S, Sandrin L (2010) Liver stiffness: a novel parameter for the diagnosis of liver disease. Hepat Med 2: 49-67.

9. Wei SC, Yang J (2016) Forcing through tumor metastasis: the interplay between tissue rigidity and epithelial-mesenchymal transition. Trends Cell Biol 26(2): 111-120.

10. Yu H, Mouw JK, Weaver VM (2011) Forcing form and function: biomechanical regulation of tumor evolution. Trends Cell Biol 21(1): 47-56. 
11. Boghaert E, Gleghorn JP, Lee KA, Gjorevski N, Radisky DC, et al. (2012) Host epithelial geometry regulates breast cancer cell invasiveness. Proc Natl Acad Sci U S A 109(48): 19632-19637.

12. Portillo-Lara R, Alvarez MM (2015) Enrichment of the cancer stem phenotype in sphere cultures of prostate cancer cell lines occurs through activation of developmental pathways mediated by the transcriptional regulator $\Delta \mathrm{Np} 63 \alpha$. PLoS One $10(6)$ : e0130118.

13. Shebanova O, Hammer DA (2012) Biochemical and Mechanical Extracellular Matrix Properties Dictate Mammary Epithelial Cell Assembly. Biotechnol J 7(3): 397-408.

14. Beck JN, Singh A, Rothenberg AR, Elisseeff JH, Ewald AJ (2013) The independent roles of mechanical, structural and adhesion characteristics of 3D hydrogels on the regulation of cancer invasion and dissemination. Biomaterials 34(37): 9486-9495.

15. Koike C, McKee TD, Pluen A, Ramanujan S, Burton K, et al. (2002) Solid stress facilitates spheroid formation: potential involvement of hyaluronan. Br J Cancer 86(6): 947-953.

16. Cheng G, Tse J, Jain RK, Munn LL (2009) Micro-environmental mechanical stress controls tumor spheroid size and morphology by suppressing proliferation and inducing apoptosis in cancer cells. PLoS One 4(2): e4632.

17. Rijal G, Li W (2016) 3D scaffolds in breast cancer research. Biomaterials 81: 135-156.

18. Araki C (1956) Structure of the agarose constituent of agar-agar. Bull Chem Soc Jpn 29: 543-544.

19. Cheng S-Y, Heilman S, Wasserman M, Archer S, Shulerac ML, Wu M (2007) A hydrogel-based microfluidic device for the studies of directed cell migration. Lab Chip 7(6): 763-769.

20. Ling Y, Rubin J, Deng Y, Huang C, Demirci U, et al. (2007) A cell-laden microfluidic hydrogel. Lab Chip 7(6): 756-762.

21. White JA, Deen WM (2002) Agarose-dextran gels as synthetic analogs of glomerular basement membrane: water permeability. Biophys J 82(4): 2081-2089.

22. Ho WY, Yeap SK, Ho CL, Rahim RA, Alitheen NB (2012) Development of multicellular tumor spheroid (MCTS) culture from breast cancer cell and a high throughput screening method using the MTT assay. PLoS One $7(9)$ : e44640.

23. Deepthi R, Bhargavi R, Jagadeesh K, Vijaya MS (2010) Rheometric studies on agarose gel - a brain mimic material. SAS Tech J 9(2): 27-30.

24. Byron ML, Variano EA (2013) Refractive-index-matched hydrogel materials for measuring flow-structure interactions. Exp Fluids 54(2): 1-6.

25. Jain A, Yang AHJ, Erickson D (2012) Gel-based optical waveguides with live cell encapsulation and integrated microfluidics. Opt Lett 37(9): 1472-1474.

26. Afrimzon E, Botchkina G, Zurgil N, Shafran Y, Sobolev M, et al. (2016) Hydrogel microstructure live-cell array for multiplexed analyses of cancer stem cells, tumor heterogeneity and differential drug response at single-element resolution. Lab Chip 16(6): 1047-1062.

27. Napolitano AP, Dean DM, Man AJ, Youssef J, Ho DN, et al. (2007) Scaffoldfree three-dimensional cell culture utilizing micromolded nonadhesive hydrogels. Biotechniques 43(4): 494-500.
28. Martowicz A, Spizzo G, Gastl G, Untergasser G (2012) Phenotypedependent effects of EpCAM expression on growth and invasion of human breast cancer cell lines. BMC Cancer 12: 501.

29. Sun Y, Mao X, Fan C, Liu C, Guo A, et al. (2014) CXCL12-CXCR4 axis promotes the natural selection of breast cancer cell metastasis. Tumour Biol 35(8): 7765-7773.

30. Lopez JI, Kang I, You W-K, McDonald DM, Weaver VM (2011) In situ force mapping of mammary gland transformation. Integr Biol (Camb) 3(9): 910-921.

31. Froehlich K, Haeger JD, Heger J, Pastuschek J, Photini SM, et al. (2016) Generation of Multicellular Breast Cancer Tumor Spheroids: Comparison of Different Protocols. J Mammary Gland Biol Neoplasia 21(3-4): 89-98.

32. Nath S, Devi GR (2016) Three-dimensional culture systems in cancer research: Focus on tumor spheroid model. Pharmacol Ther 163: 94-108.

33. do Amaral JB, Urabayashi MS, Machado-Santelli GM (2010) Cell death and lumen formation in spheroids of MCF-7 cells. Cell Biol Int 34(3): 267-274.

34. do Amaral JB, Rezende-Teixeira P, Freitas VM, Machado-Santelli GM (2011) MCF-7 cells as a three-dimensional model for the study of human breast cancer. Tissue Eng Part C Methods 17(11): 1097-1107.

35. (2010) The International Agency for Research on Cancer. In: Bosman FT, et al. (Eds.), WHO Classification of Tumours of the Digestive System. ( $4^{\text {th }}$ edn). World Health Organization, Geneva.

36. Friedl P, Locker J, Sahai E, Segall JE (2012) Classifying collective cancer cell invasion. Nat Cell Biol 14(8): 777-783.

37. Zavyalova MV, Perelmuter VM, Vtorushin SV, Denisov EV, Litvyakov NV, et al. (2013) The presence of alveolar structures in invasive ductal NOS breast carcinoma is associated with lymph node metastasis. Diagn Cytopathol 41(3): 279-282.

38. Denisov EV, Litviakov NV, Zavyalova MV, Perelmuter VM, Vtorushin SV, et al. (2014) Intratumoral morphological heterogeneity of breast cancer: neoadjuvant chemotherapy efficiency and multidrug resistance gene expression. Sci Rep 4: 4709.

39. Dabrowska-Piaskowska K (1959) Observations on the histoformative capacities of tumor cells dissociated by digestion with trypsin. Exp Cell Res 16(2): 315-323.

40. Hyun K-A, Koo G-B, Han H, Sohn J, Choi W, et al. (2017) Epithelial-tomesenchymal transition leads to loss of EpCAM and different physical properties in circulating tumor cells from metastatic breast cancer. Oncotarget 7(17): 24677-24687.

41. Olea N, Villalobojs M, de Almodóvar MR, Pedraza V (1992) MCF-7 breast cancer cells grown as multicellular spheroids in vitro: effect of 17ß-estradiol. Int J Cancer 50(1): 112-117.

42. Markovitz-Bishitz Y, Tauber Y, Afrimzon E, Zurgil N, Sobolev M, et al. (2010) A polymer microstructure array for the formation, culturing, and high throughput drug screening of breast cancer spheroids. Biomaterials 31(32): 8436-8444.

43. Bozorgi A, Khazaei M, Khazaei MR (2015) New Findings on Breast Cancer Stem Cells: A Review. J Breast Cancer 18(4): 303-312. 